\title{
DISAGGREGATION OF HOUSE ELECTRICITY CONSUMPTION USING PARTICLE SWARM OPTIMIZATION
}

\author{
TAN RUI LIN, NURUL NAJEEHAH RAZALI AND MUHAMAD ZALANI DAUD*
}

Renewable Energy and Power Research Interest Group (REPRIG), Faculty of Ocean Engineering Technology and Informatics, Universiti Malaysia Terengganu, 21030 Kuala Nerus, Terengganu, Malaysia.

\begin{abstract}
This paper presents disaggregation of appliances' energy consumption for a residential house using Particle Swarm Optimization (PSO). The disaggregation process provides useful information about the time and duration of each appliance on when they are switched on, which can contribute to close monitoring of appliance activities in a house. Firstly, the data of aggregated electricity loads comprising six different appliances is measured and plotted based on total power $\left(P_{\text {total }}\right)$ over time. The data is then formulated based on combinatorial optimization (CO) problem which represents the load disaggregation model that contains the sum power of all six appliances when they are switched on. The CO problem is solved by using PSO by setting up the population of particles to represent each appliance. The random number of each population is generated based on appliance's power state when switched on, where the total appliances power $\left(P_{a p p}\right)$ is represented as the sum of all six particles data. Thus, by using the known value of appliance on state as optimization boundary parameters, the CO problem is evaluated by PSO following the Integral Squared Error (ISE) minimization problem of $P_{\text {total }}$ and $P_{a p p}$. The optimization results give good convergence criteria with accurate percentage of time taken for every appliance in use. [206 words]
\end{abstract}

Keywords: Energy efficiency, load disaggregation, heuristic optimization, Particle Swarm Optimization, non-intrusive load monitoring.

\section{Introduction}

In 2000 to 2015, Malaysian household electricity consumption per capita increased from 484.1 $\mathrm{kWh}$ to $935.4 \mathrm{kWh}$, which means that the usage of electric energy had increased up to $93.22 \%$ for the past 15 years and is expected to double from 2010 to 2050 (Larcher \& Tarascon, 2015). When electricity energy demand increases, the amount of energy waste also increases. One of the factors that has contributed to this issue is the lack of awareness among the users. Many users do not know that their electric appliance is still consuming electric power in standby mode. Although some of the appliances do not have the standby mode, when connected to the power supply without performing any operation small amounts of energy are still lost (Ajay-D-Vimal Raj, Sudhakaran \& Philomen-D-Anand Raj, 2009).
Based on this, smart meter is introduced to overcome this problem. It is a kind of advanced energy meter which can calculate and present the amount of electric energy usage from time to time to the users. Besides, it can record the energy consumption value every day at low resolutions in database or cloud compared to the traditional electric meter (Faustine, Mvungi, Kaijage \& Michael, 2017) has led to the rise of Non-Intrusive Load Monitoring (NILM. This enables the user to know how much electric energy they have consumed and which appliance absorbs more energy. Information from the smart meter can be monitored from their computer, laptop or phone at anytime, anywhere. From there, it provides the mechanism for the user to closely monitor their usage and be aware of the importance of saving energy. 
Currently, there is an emerging technology that can be used to disaggregate the electricity loads to its individual components. Energy disaggregation is a computational technique for estimating appliance-by-appliance energy consumption from a house energy meter signal. The state-of-the art infrastructure for this kind of load disaggregation concept is called NonIntrusive Load Monitoring (NILM) which was first introduced by Hart (1992). Using NILM, a disaggregation algorithm, these data can be broken up into individual appliance consumption patterns that describe its total energy used, usage frequency and time. Each appliance is identified and classified depending on the load signature of every appliance. Figure 1 shows the first general concept of NILM by Hart. Based on the individual appliance usage data, further actions can be taken such as awareness campaigns to consumers as well as technological intervention to improve the energy efficiency (Kelly \& Knottenbelt, 2015).

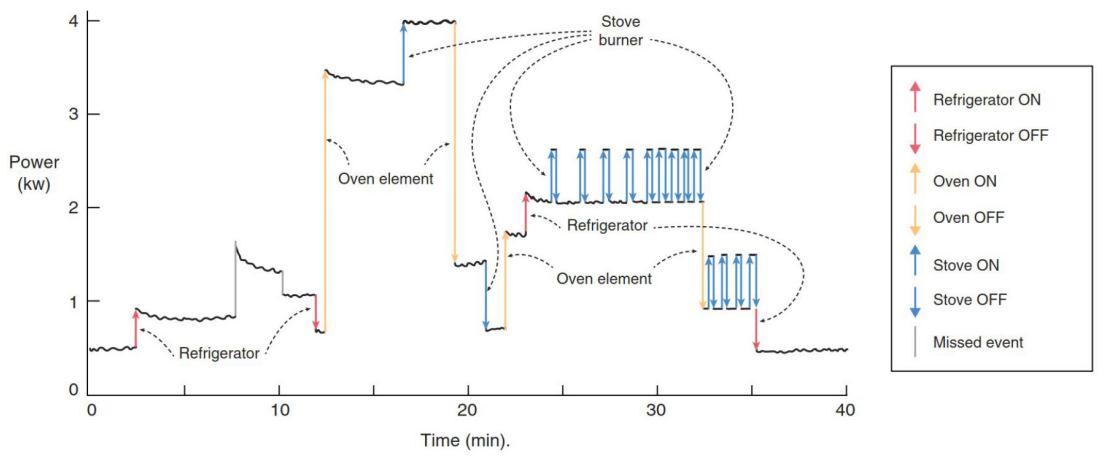

Figure 1: NILM concept for load disaggregation proposed by George W. Hart (Hart, 1992)

In general, NILM system is divided into optimization and pattern recognition base algorithms (Zoha, Gluhak, Imran \& Rajasegarar, 2012). Most of the optimization method problem is solved by combinatorial optimization (CO) as the following Equation (Batra et al., 2014).

$$
\left[x_{t}^{1}, x_{t}^{2}, x_{t}^{3} \mathrm{~K} \quad \mathrm{~K} \quad \mathrm{~K} \quad x_{t}^{N}\right]=\arg \min [|P t-\bar{P} t|] \quad \text { (Equation 1) }
$$

where,

$X_{t}=$ the optimum operating state of each appliance

$P_{t}=$ power measured at time, $\mathrm{t}$

$\bar{P}=$ total power measured at time, $\mathrm{t}$

$N=$ Number of appliances

In 2014, (Batra et al., 2014) develop an open source Non-intrusive Load Monitoring Toolkit (NILMTK) to compare the energy disaggregation algorithms in a reproducible manner. It was used to solve non-standard data sets for evaluation of algorithms and different accuracy metrics for performance evaluation. The disaggregation algorithm was improved by (Kelly, Gate \& Knottenbelt, 2015) which introduced three methods of deep learning namely recurrent neural network, denoising autoencoders and neural network which regresses the start time, end time and average power demand of each appliance activation. This work was dedicated to solve the pattern recognition-based problems. Another research from (Egarter \& Elmenreich, 2015) highlights several meta-heuristic approaches as solutions for NILM system -based optimization. The technique based on evolutionary algorithm, differential evolution, simulated annealing, cuckoo search algorithm and firefly optimization has been investigated. The problem was to solve the $\mathrm{CO}$ that relates to the Knapsack problem. The tested optimization algorithms show good results for ON/OFF based electrical appliances when power drawn by the appliance is different and easy to separate, 
and besides that, (Barbarosou et al., 2017) to identify and classify low frequency power of appliances based on true power and reactive power. There are four algorithms such as Decision Tree, Nearest Neighbor, Discriminant Analysis, and multilayer Feed-forward Neural Network classifier to be tested. However, only multilayer Feed-forward Neural Network shows high accuracy of $98 \%$ for disaggregating the loads. In this paper, a similar approach of using heuristic methods is proposed that is by using Particle Swarm Optimization (PSO). Here, the aggregated electricity loads data is measured and plotted based on the total power over time. Then, the problem is formulated based on the $\mathrm{CO}$ problem and solved by PSO. The accurate load disaggregation strategy is achieved when the objective function is evaluated to its best fitness and good convergence criteria of PSO.

\section{Materials and Methods}

\section{Data Acquisition}

The conventional method of knowing the total consumption of electricity of a house is by referring to the amount of power usage over one month. The energy consumption can be understood by referring to the electricity bill. However, the electricity bill information provided to the consumer was not presented in detail, which means, the user would not really know which appliance consumed most and vice versa. Therefore, the smart meter is introduced as a tool that uses the NILM disaggregation algorithm to carry out energy disaggregation strategy. For the NILM methodology to work, it is desirable to carry out data logging of energy which measures the voltage, current and power in real time. Normally, a data acquisition module is used to acquire aggregated load measurement at the energy meter at an adequate rate so that distinctive load patterns can be identified (Zoha et al., 2012). In this case, the data is collected every 90 seconds for 12.5 hours.

\section{Feature Extraction}

The collected data is extracted to reveal more information about appliance usage. Obtaining the features of appliances can be done by detection of events such as appliance state transitions. The features can be classified into three types, steady state, transient state and non-traditional features. Steady state features refer to the steady-state operation of an appliance, only the real power and reactive power value is needed to detect the change state events operation of the appliance. Compared to steady state feature, transient state feature derived from the transient state operation of an appliance is less overlapping between appliances than the steady state feature. The non-traditional features are the result of the other two kinds of characteristics such as time of the day, ON/OFF distribution, use frequency of an appliance and the correlation of usage of multiple appliances. There are six types of appliances to be studied in this case.

\section{Inferences and Learning}

The result of applied load identification algorithms is a technique to determine the appliances that are running at a given time which can be divided into supervised, semi-supervised and unsupervised techniques. The supervised methods require individual appliance data to be trained to classify the appliances which are working at each moment and the algorithm works effectively. Semi-supervised techniques need to train some data at the beginning of the process to perform the classification, while the unsupervised method is capable of learning from the data collected without previous training data. All methods are highly explored nowadays because of low setup costs, non-intrusiveness and short training phase for load identification algorithms.

\section{Appliance Classification and Load Disaggregation}

After completing the load identification, the next step is to assign the total consumption among the identified loads, then detail all the information on the amount of consumption provided by each appliance. The information is processed in the form of statistics which can be read and easily understood by the users. 


\section{Problem Formulation of NILM}

From equation 1 , the total power measured at time $t$ for each appliance, $P t$ for all $N$ appliances including measurement noise, $e_{t}$ can be expressed by using equation 2 . Assuming that measurement error can be negligible and all the appliances are two-state devices, the power consumption of the $i$-th appliance at time $t$ can be described as equation 3 (Hart, 1992).

$$
\begin{gathered}
P t=\sum_{i=1}^{N} p_{t}^{i}+e_{t} \\
p_{t}^{i}=p^{i} x_{t}^{i} \quad i=1,2 \mathrm{~K} \mathrm{KN}
\end{gathered}
$$

where,

$e_{t}=$ noise measure

$p_{t}^{i}=$ power in operating state

$x_{t}^{i=}$ the state of appliance $i$ at time $t$ whether in

ON/OFF state

$p^{i}=$ operating power of appliances

Therefore, the load disaggregation is transformed into a mathematical optimization problem to define the optimum operating state of each appliance. This is the combinatorial optimization (CO) problem, which has been discussed in the traditional load disaggregation model of NILM which can be solved by using an intelligent algorithm such as PSO.

\section{Solving the Optimization Problem Using PSO}

PSO is an optimization algorithm based on swarm intelligence introduced by (Eberhart \& Kennedy, n.d.). PSO algorithms imitate the navigation and foraging of a flock of birds or a school of fishes. Particles are a potential solution in PSO which have "flown" through the problem space by following the present optimum particles. The particles form swarms and travel within the search space to find the simplest solution to reach the target. Each particle in search space will adjust the flying track according to flying experience by its own or other particles. Each particle always keeps track of the best solution and knows as personal best (pbest) and global best (gbest). Each particle modifies the position according to current position, current velocity, distance between current position and pbest and the distance of current position and gbest.

Besides, PSO has a much more profound intelligent background, easy to implement, robustness to control parameters and computation efficiency compared with other existing heuristic algorithms such as genetic algorithms in a continuous problem. Normally, PSO can be applied to non-differentiable, nonlinear, huge search space problems and will provide better results (Russell C. Eberhart, 2001). Figure 2 shows the flow on how the PSO work step by step. 


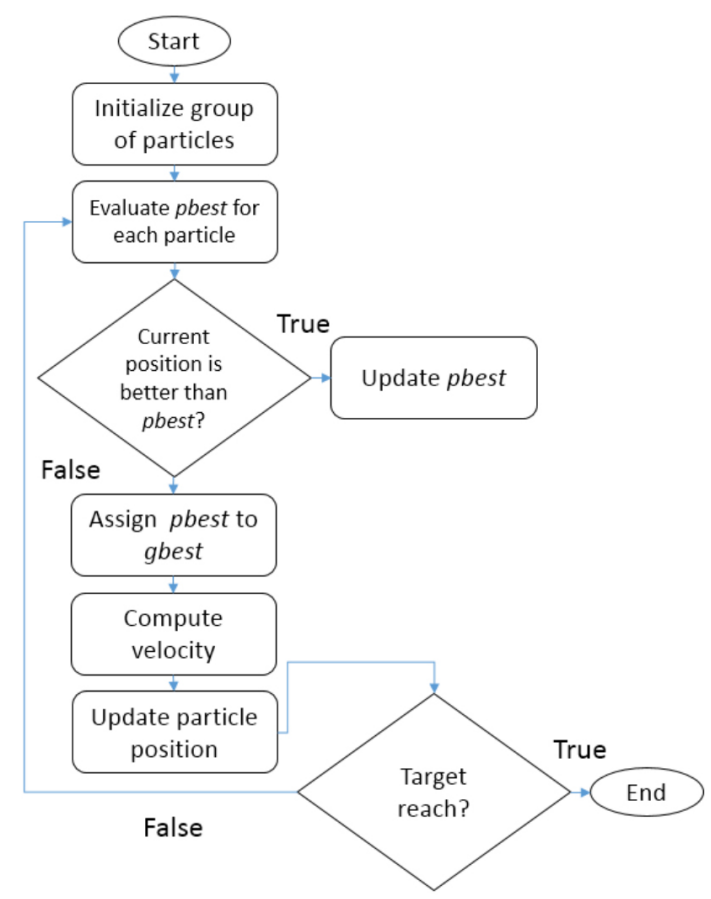

Figure 2: Flowchart on how the PSO works

In this case, the raw data of 6 actively switching ON/OFF appliances is analysed as shown in Table 1. The minimum power and maximum power of each appliance during OFF/ ON state are shown in Table 1 and are set as the lower boundary and upper boundary values for PSO.

Table 1: Power range of each appliance

\begin{tabular}{ccc}
\hline Appliance No. & Minimum Power (W)/Lower Boundary & Maximum Power (W)/Upper Boundary \\
\hline 1 & 0 & 35 \\
2 & 0 & 55 \\
3 & 0 & 130 \\
4 & 0 & 3000 \\
5 & 0 & 2800 \\
6 & 0 & 2400 \\
\hline
\end{tabular}

According to the lower boundary and upper boundary power limit of each appliance, the PSO operator will randomly generate the population for each appliance. Each appliance generated 500 sets of data and declared as particle 1 to particle 6. From this, a total of 3000 sets of data are added and represented as $P_{a p p}$ for further process. The main task of the PSO operator is to carry out error minimization based on the integral squared error (ISE) fitness function. Therefore, the objective function considered in this operation implemented in Matlab is shown as equation 4. The fitness of each particle is evaluated using PSO so that in the end the stopping criterion or the iteration is achieved with a good convergence criteria (Batra et al., 2014). 


$$
I S E=\sum_{i=1}^{6}\left[\left(P_{a p p_{i}}-P_{\text {total }_{i}}\right)^{2}\right]
$$

(Equation 4)

Basically, in every iteration, PSO will create an initial population which is uniformly distributed over the space. Each particle's position is evaluated according to the objective function. Each particle updates its current position for every iteration if the current position is better than the previous position. At the same time, the particles will determine its best particle and always update its velocities. The velocities of the particle will be updated according to equation 5 (Russell C. Eberhart, 2001).

$$
v_{i}(t+1)=w v_{i}(t)+r_{1} C_{1}\left(\mathrm{P}_{i}^{p}(t)-\mathrm{x}_{i}\right)+r_{2} C_{2}\left(\mathrm{P}_{i}^{g}(t)-\mathrm{x}_{i}\right)
$$

(Equation 5)

where,

$w=$ Inertia

$x_{i}=$ Position of the particle

$v_{i}=$ Velocity of the particle

$C_{1}=$ Cognitive parameter

$C_{2}=$ Social parameter

$t=$ Generation

$r=$ Random number

$\mathrm{P}_{i}^{p}=$ pbest of a particle

$\mathrm{P}_{i}^{g}=$ gbest of a particle
The velocity of the particles depends on the inertia, local search and also global search. Inertia helps to make sure the particles move in the same direction and with the same velocity. Local search influence will improve the individual position of a particle while global best will make the particles follow the best neighbour direction. The error minimization problem will achieve its best convergence criteria when the sum of power of all 6 appliances is nearly the same as the raw data power.

\section{Results and Discussion}

The result of average data, total usage time and percentage of usage time for each appliance is discussed in this section.

\section{Objective Function Graph}

Figure 3 presents a correlation between the fitness value and maximum iteration which is 5000 iterations. As shown in Figure 3, the graph is in a converge shape as it met with the expectations to get objective functions that have a minimum error. The inertial weight of the parameter function is to improve the convergence precision. It clearly shows from the figure that the process converges at the first 500 iterations.

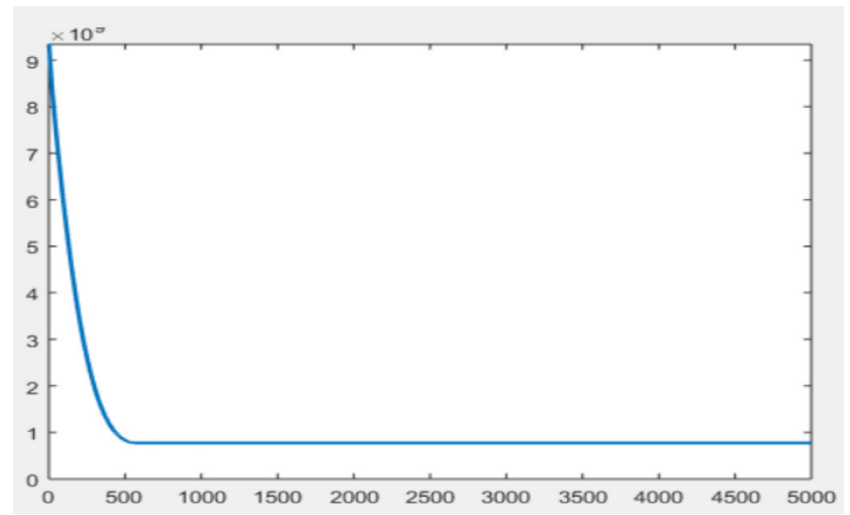

Figure 3: Graph for minimum error 


\section{Average Value for Each Appliance}

From the result in Figure 3, the average data for appliance 1 to appliance 6 are plotted in Figure 4 and discussed further in this section.
As in Figure 4, the average data for each appliance over the whole 500 plotted data is shown at y-axis. The average data value for appliance 1 is 13.2 , appliance 2 is 5.5 and 38.6 for appliance 3. Besides, average data value of appliance 4, appliance 5 and appliance 6 is $981.0,146.8$ and 301.5 , respectively.

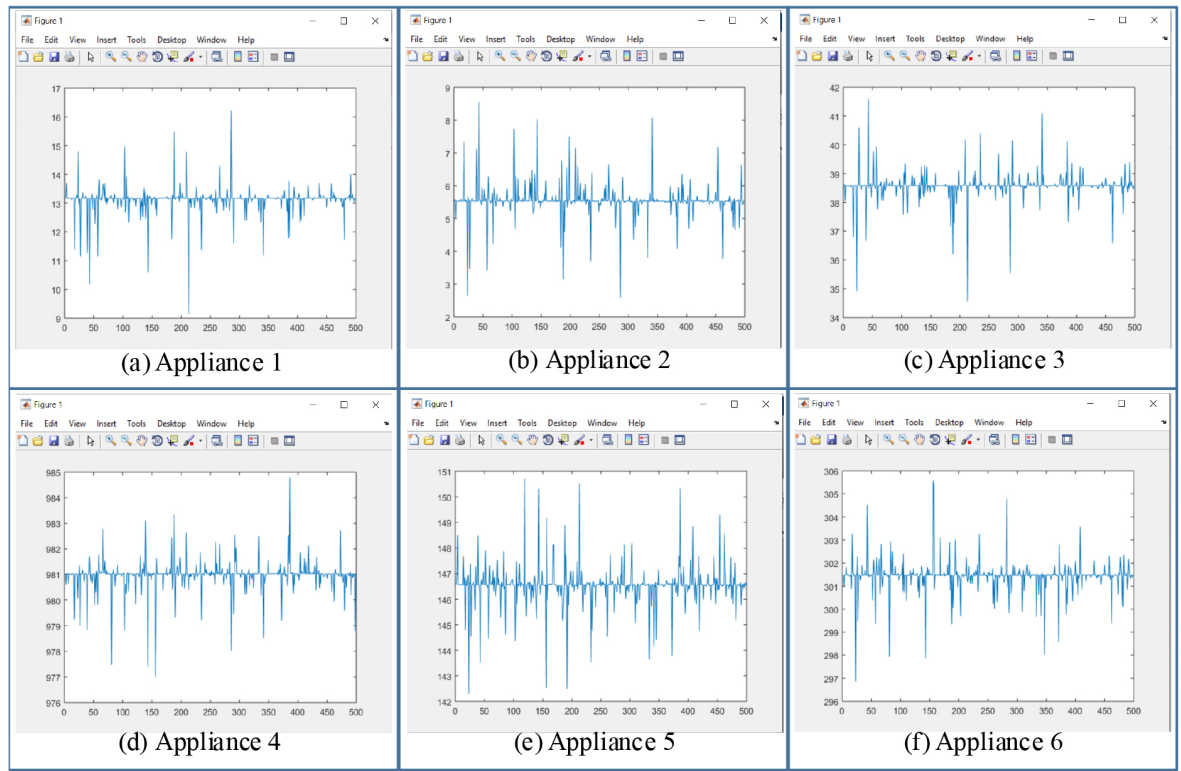

Figure 4: Average data for appliance 1 to appliance 6

The energy consumed by each appliance is the area under the graph. Thus, to get the area under the plotted data the following equation applies:

$$
\text { Area }=\text { Width } x \text { Height }
$$

(Equation 6)

where,

Width $=$ Amount of plotted data in 12.5 hours

Height $=$ Average data of each appliance in watts

As a result, area under the graph in Figure 4 for the appliance 1 is 6600, appliance 2 and appliance 3 is 2750 and 19300 , respectively. In addition, appliance 4, appliance 5 and appliance 6 , the value under the graph for each appliance is 4905000,734000 and 150750 , respectively.

\section{Total Time Consumption for Each Appliance}

From the total power graph, each appliance has a different power consumption. For example, appliance 1 is $35 \mathrm{~W}$, appliance 2 equals 55 $\mathrm{W}$, appliance 3 consumes $130 \mathrm{~W}$, appliance 4, appliance 5 and appliance 6 consume $3000 \mathrm{~W}$, $2800 \mathrm{~W}$ and $2400 \mathrm{~W}$, respectively. To identify the total consumption time, T of each appliance, the value of area under the graph and maximum power consumption value of each appliance substitute in equation 7 . Furthermore, the results for the average data, power consumption and total time power consumption of each appliance are shown in Table 2. Moreover, the usage time of each appliance in ON state was also calculated to transform in percentage and described in pie chart as shown as in Figure 5.

$$
T=\frac{\text { Area }}{\text { Area }} \times 90 s
$$

(Equation 7) 
where

$P=$ Maximum power consumption of appliance

$90 s=$ Actual data collect every 90 seconds

Table 2: Average data and usage time for each appliance

\begin{tabular}{lcccccc}
\hline \multicolumn{1}{c}{ Appliance } & $\mathbf{1}$ & $\mathbf{2}$ & $\mathbf{3}$ & $\mathbf{4}$ & $\mathbf{5}$ & $\mathbf{6}$ \\
\hline Average data value & & & & & & \\
Maximum power consumption & 13.20 & 5.50 & 38.60 & 981.00 & 146.80 & 301.50 \\
(Watt) & 35.00 & 55.00 & 130.00 & 3000.00 & 2800.00 & 2400.00 \\
Total consumption time (Second) & 16971.00 & 4500.00 & 13361.00 & 14715.00 & 2359.00 & 5653.00 \\
Total consumption time (Minute) & 282.85 & 75.00 & 222.68 & 245.25 & 39.32 & 94.22 \\
\hline
\end{tabular}

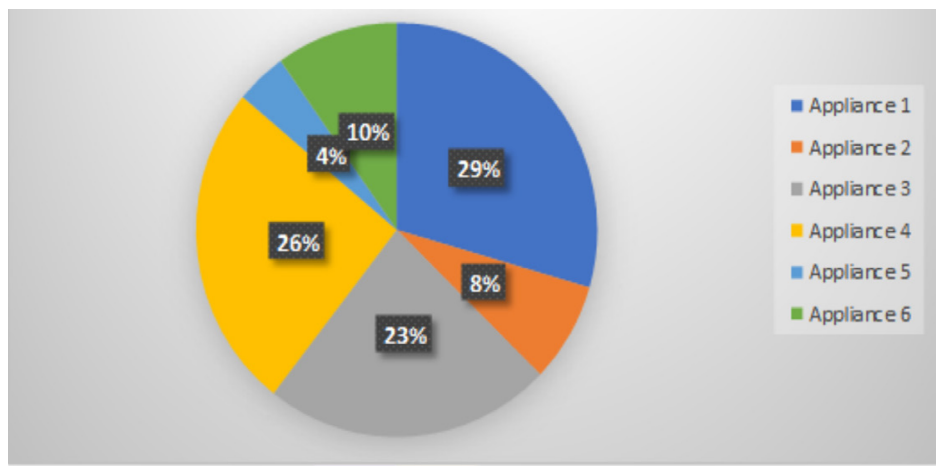

Figure 5: Percentage of time taken for appliance in use

\section{Conclusion}

This paper has presented a method based on heuristic optimization, using PSO to solve the optimization problem arising in a load disaggregation of electrical appliances. From the results, the PSO operator can provide good convergence criteria where from 5000 number of iterations, the system can converge at just 500 iterations. The results also show that the total loads can be disaggregated effectively where all six appliances' operation state can be estimated and classified according to how they operate and how much energy has been consumed. This will help the user to identify which appliance consumes the power most and can be a guide for a proper management of appliance usage at home.

\section{Acknowledgements}

The authors would like to acknowledge Universiti Malaysia Terengganu, Malaysia for the financial support of this research. This research is supported by UMT under the TAPERG grant scheme, Vot. No. 55221.

\section{References}

Ajay-D-Vimal Raj, P., Sudhakaran, M., \& Philomen-D-Anand Raj, P. (2009). Estimation of standby power consumption for typical appliances. Journal of Engineering Science and Technology Review, 2(1), 71-75. https://doi. org/10.1016/j.jhevol.2008.08.011 
Barbarosou, M., Papageorgas, P., Corre, L., Hamid, O., Barbarosou, M., Papageorgas, P., Salame, C. (2017). Automatic recognition of electric loads analyzing the characteristic parameters of the consumed electric power through a Non-Intrusive Monitoring methodology. Energy Procedia, 119, 742-751. https://doi.org/10.1016/j. egypro.2017.07.137

Batra, N., Kelly, J., Parson, O., Dutta, H., Knottenbelt, W., Rogers, A., ... \& Srivastava, M. (2014, June). NILMTK: an open source toolkit for non-intrusive load monitoring. In Proceedings of the 5th international conference on Future energy systems (pp. 265-276).

Eberhart, R., \& Kennedy, J. (1995, October). A new optimizer using particle swarm theory. In MHS'95. Proceedings of the Sixth International Symposium on Micro Machine and Human Science (pp. 39-43). Ieee.

Egarter, D., \& Elmenreich, W. (2015, November). Load disaggregation with metaheuristic optimization. In Energieinformatik (pp. $1-12)$.

Faustine, A., Mvungi, N. H., Kaijage, S., \& Michael, K. (2017). A Survey on NonIntrusive Load Monitoring Methodies and Techniques for Energy Disaggregation Problem. Retrieved from http://arxiv.org/ abs/1703.00785
Hart, G. W. (1992). Nonintrusive appliance load monitoring. IEEE, 80(12), 1870-1891.

Kelly, J., \& Knottenbelt, W. (2015, November). Neural nilm: Deep neural networks applied to energy disaggregation. In Proceedings of the 2nd ACM International Conference on Embedded Systems for Energy-Efficient Built Environments (pp. 55-64). https://doi. org/10.1145/2821650.2821672

Kelly, J., \& Knottenbelt, W. (2015). Does disaggregated electricity feedback reduce domestic electricity consumption? A systematic review of the literature.

Larcher, D., \& Tarascon, J. M. (2015). Towards greener and more sustainable batteries for electrical energy storage. Nature Chemistry, 7(1), 19-29. https://doi.org/10.1038/ nchem. 2085

Russell C. E., \& Yuhui. S. (2001). Particle swarm optimization: developments, applications and resources. In Proceedings of the 2001 congress on evolutionary computation (IEEE Cat. No. 01TH8546) (Vol. 1, pp. 81-86). IEEE. https://doi.org/10.1109/ CEC.2001.934374

Zoha, A., Gluhak, A., Imran, M. A., \& Rajasegarar, S. (2012). Non-Intrusive Load Monitoring Approaches for Disaggregated Energy Sensing: A Survey. Sensors, 16838 16866. https://doi.org/10.3390/s121216838 
